\title{
Wilson Chiral Perturbation Theory for twisted mass QCD at NLO
}

\section{Satoru Ueda*}

Graduate School of Pure and Applied Sciences

University of Tsukuba

Tsukuba, Ibaraki 305-8571

Japan

E-mail: sueda@het.ph.tsukuba.ac.jp

\section{Sinya Aoki}

Graduate School of Pure and Applied Sciences

University of Tsukuba

Tsukuba, Ibaraki 305-8571

Japan, and

Riken BNL Research Center

Brookhaven National Laboratory

Upton, New York 11973

USA

E-mail: saoki@het.ph.tsukuba.ac.jp

We investigate the quark mass dependence of pion sectors in the twisted mass lattice QCD, using the Wilson Chiral Perturbation Theory (WChPT). In order to consider the small quark mass regime such that $m_{q} \sim a^{2} \Lambda^{3}$, we include $O\left(a^{2}, a m\right)$ terms at the leading order (LO), which induce a non-trivial phase structure at the tree level. At the next leading order (NLO), due to the presence of the twisted mass, not only pion masses but also a vacuum expectation of the neutral pion have divergences, which must be removed by local counter terms at the NLO. We demonstrate that cancellations of these divergences can be consistently performed within a framework of the WChPT. As an interesting application of the NLO calculation, we derive the twisted quark mass dependence of the pion mass at the maximal twist where the PCAC quark mass vanishes.

The XXVI International Symposium on Lattice Field Theory

July 14-19 2008

Williamsburg, Virginia, USA

\footnotetext{
* Speaker.
} 


\section{Introduction}

The twisted mass lattice QCD (tmLQCD) [1, 2] has several advantages for numerical simulations lattice QCD (LQCD) with other fermion formulations. One of its advantages is an automatic $O(a)$ improvement at the maximal twist: Physical observables of the tmLQCD are free from $O(a)$ lattice artifact if the twist angle becomes maximal by tuning untwisted quark mass parameter [5 6. Utilizing this and other advantages of the tmLQCD for reductions of computational costs, the European twisted mass (ETM) collaboration was able to carry out full QCD simulations at the lattice spacing $a \lesssim 0.1 \mathrm{fm}$ and the charged pion mass $m_{\pi} \sim 300 \mathrm{MeV}$ [3, 4].

The Wilson chiral perturbation theory (WChPT) is an important tool of LQCD with Wilsontype quark actions, where $O(a)$ lattice artifacts potentially exist. In the (untwisted) Wilson fermion case, the WChPT can predict an existence of the parity-flavor symmetry breaking phase transition [7], which is caused by the $O\left(a^{2}\right)$ chiral breaking term[8]. Furthermore the quark mass dependence of pion mass and decay constant have been calculated at the next to leading order (NLO) of the WChPT in the small quark mass region 9 .

In the case of the tmLQCD, several investigations have been performed by the WChPT[10, 11,12]. However there are no NLO calculations in the small quark mass region where $O\left(a^{2}\right)$ terms becomes important so that they must be treated as LO terms. In this study we therefore perform NLO calculation of the WChPT in the small quark mass region and investigate the quark mass dependence of pion masses at the NLO.

As an interesting application of the NLO calculation, we derive the twisted quark mass dependence of the pion mass at the maximal twist which is defined through the PCAC quark mass and has been employed by the ETM collaboration for numerical simulations [3].

\section{Power counting and Lagrangian}

If a size of the quark mass $m$ is comparable to the lattice spacing a, i.e. $m \sim a \Lambda^{2}$ where $\Lambda$ is a typical scale of QCD such as $\Lambda_{\overline{\mathrm{MS}}}$. In this case, the power counting of the WChPT is given by

$$
\mathrm{LO} \sim O\left(p^{2}, m, a\right), \quad \mathrm{NLO} \sim \mathrm{LO}^{2} \sim O\left(p^{4}, m^{2}, a^{2}, a p^{2}, a m\right),
$$

and $O(a)$ contributions at the $\mathrm{LO}$ can be absorbed to $O(m)$ terms, so that the WChPT is almost identical to the ChPT at this order with $\tilde{m}=m+O(a)$.

As the quark mass decreases further so that $m \sim a^{2} \Lambda^{3}, O\left(a^{2}\right)$ terms become important and responsible for the parity-flavor breaking phase transition. Since we are interested in such a small quark mass region, we employ the following power counting[9], where the $O\left(a^{2}, a m\right)$ terms are included at the LO, and are denoted by sub leading order (SLO) terms to be distinguished from LO terms.

$$
\begin{aligned}
\mathrm{LO} & \sim O\left(p^{2}, \tilde{m}\right) \sim O(M), \quad \mathrm{SLO} \sim O\left(a^{2}, a M\right), \\
\mathrm{NLO} & \sim \mathrm{LO}^{2}, \mathrm{LO} \times \mathrm{SLO} \sim O\left(M^{2}, a^{2} M, a M^{2}\right),
\end{aligned}
$$

where $M=p^{2}$ or $\tilde{m}$. Here we neglect the $\mathrm{SLO}^{2}$ terms. Therefore the above power counting is valid for $\mathrm{LO}>\mathrm{SLO}$ but not for $\mathrm{LO} \simeq \mathrm{SLO}$. 
We consider the WChPT for $N_{f}=2$ tmLQCD. In our power counting, the LO and SLO chiral Lagrangian becomes

$$
\begin{aligned}
\mathscr{L}_{\mathrm{LO}} & =\frac{f^{2}}{4}\left\langle\partial_{\mu} \Sigma \partial_{\mu} \Sigma^{\dagger}\right\rangle-\frac{f^{2}}{4}\left\langle\Sigma M^{\dagger}+M \Sigma^{\dagger}\right\rangle-\frac{f^{2}}{4}\left\langle\Sigma \hat{a}^{\dagger}+\hat{a} \Sigma^{\dagger}\right\rangle \\
& +W_{45}\left\langle\partial_{\mu} \Sigma \partial_{\mu} \Sigma^{\dagger}\right\rangle\left\langle\left(\Sigma-\Sigma_{0}\right) \hat{a}^{\dagger}+\hat{a}\left(\Sigma-\Sigma_{0}\right)^{\dagger}\right\rangle \\
& -W_{68}\left\langle\Sigma M^{\dagger}+M \Sigma^{\dagger}\right\rangle\left\langle\Sigma \hat{a}^{\dagger}+\hat{a} \Sigma^{\dagger}\right\rangle-W_{68}^{\prime}\left\langle\Sigma \hat{a}^{\dagger}+\hat{a} \Sigma^{\dagger}\right\rangle^{2},
\end{aligned}
$$

where $\langle\ldots\rangle$ means the trace over the flavor indices, the quark mass matrix becomes $M=2 B_{0}(m+$ $\left.i \tau^{3} \mu\right)$ with $\tau^{a}$ being an SU(2) Pauli matrix for flavor space, and the lattice spacing matrix is denoted as $\hat{a}=2 W_{0} a$. Parameters $f, B_{0}, W_{0}, W_{45,68}, W_{68}^{\prime}$ are the low energy constants, and their dimensions are $[f]=\left[B_{0}\right]=1,\left[W_{0}\right]=3$, and $\left[W_{45,68}\right]=\left[W_{68}^{\prime}\right]=0$. Because of the twisted mass term and the lattice spacing, $\Sigma$ has non-zero vacuum expectation value $\Sigma_{0}$, and is expanded in terms of pion fields as

$$
\Sigma=\Sigma_{0}^{1 / 2} \exp \left[i \pi_{a} \tau^{a} / f\right] \Sigma_{0}^{1 / 2}, \quad \Sigma_{0}=\exp \left[i \phi \tau^{3}\right]
$$

Replacing the untwisted mass $m$ by the shifted mass $2 B_{0} \tilde{m}=2 B_{0} m+2 W_{0} a$ and the mass matrix $M$ by $\tilde{M}=2 B_{0}\left(\tilde{m}+i \mu \tau^{3}\right)$, we rewrite the Lagrangian as

$$
\begin{aligned}
\mathscr{L}_{\mathrm{LO}} & =\frac{f^{2}}{4}\left[1+\frac{c_{0} a}{4}\left\langle\Sigma+\Sigma^{\dagger}-\left(\Sigma_{0}+\Sigma_{0}^{\dagger}\right)\right\rangle\right]\left\langle\partial_{\mu} \Sigma \partial_{\mu} \Sigma^{\dagger}\right\rangle \\
& -f^{2}\left[\frac{2 B_{0} \tilde{m}}{4}\left\langle\Sigma+\Sigma^{\dagger}\right\rangle-\frac{2 B_{0} \mu}{4}\left\langle i\left(\Sigma-\Sigma^{\dagger}\right) \tau^{3}\right\rangle-\frac{c_{2} a^{2}}{16}\left\langle\Sigma+\Sigma^{\dagger}\right\rangle^{2}\right. \\
& \left.+\tilde{c}_{2} a\left(\frac{2 B_{0} \tilde{m}}{16}\left\langle\Sigma+\Sigma^{\dagger}\right\rangle^{2}-\frac{2 B_{0} \mu}{16}\left\langle\Sigma+\Sigma^{\dagger}\right\rangle\left\langle i\left(\Sigma-\Sigma^{\dagger}\right) \tau^{3}\right\rangle\right)\right]
\end{aligned}
$$

where

$$
c_{0}=32 W_{45} \frac{W_{0}}{f^{2}}, \quad c_{2}=-64\left(W_{68}^{\prime}-W_{68}\right) \frac{W_{0}^{2}}{f^{2}}, \quad \tilde{c}_{2}=32 W_{68} \frac{W_{0}}{f^{2}},
$$

with $\left[c_{0}\right]=1,\left[c_{2}\right]=4,\left[\tilde{c}_{2}\right]=1$ as mass dimensions of parameters.

\section{Leading order analysis}

\subsection{Gap equation at the LO}

In order to determine the phase structure from the vacuum expectation value $\Sigma_{0}$, we derive the gap equation for $\Sigma_{0}$ as

$$
2 B_{0} \tilde{m} \sin \phi-\left(c_{2} a^{2}-\tilde{c}_{2} a 2 B_{0} \tilde{m}\right) \sin 2 \phi=2 B_{0} \mu \cos \phi+\left(\tilde{c}_{2} a 2 B_{0} \mu\right) \cos 2 \phi .
$$

The same gap equation with $\tilde{c}_{2}=0$ was analyzed in Ref.[11]. In Fig[1]a) we plot the phase structure in a $(\tilde{m}, \mu)$ plane (multiplied by $B_{0} /\left|c_{2} a^{2}\right|$ for $c_{2}<0$ ) and $\tilde{c}_{2}=0$. There is a first order phase transition line between $(0,-1)$ and $(0,1)$ : If $\tilde{m}$ goes from positive region to negative regions at $-1<B_{0} \mu /\left|c_{2} a^{2}\right|<1, \cos \phi$ also changes its sign. In Fig $\left.1 \mathrm{~b}\right) \cos \phi$ is plotted as a function of $B_{0} \tilde{m} /\left|c_{2} a^{2}\right|$ at the positive end point of the first order phase transition line for $\tilde{c}_{2}=0$ case ( the blue line between $(-3,1)$ and $(3,1)$ in Fig, 1a) ). In the case of $\tilde{c}_{2}=0, \cos \phi$ continuously but rapidly changes around $B_{0} \tilde{m} /\left|c_{2} a^{2}\right|=0$ and becomes exactly zero at $B_{0} \tilde{m} /\left|c_{2} a^{2}\right|=0$ ( black solid line ). As $\tilde{c}_{2}$ increases from zero, $\cos \phi$ becomes zero at more negative value of $B_{0} \tilde{m} /\left|c_{2} a^{2}\right|$, as shown by red dotted and green dashed lines in Fig 1 b). 


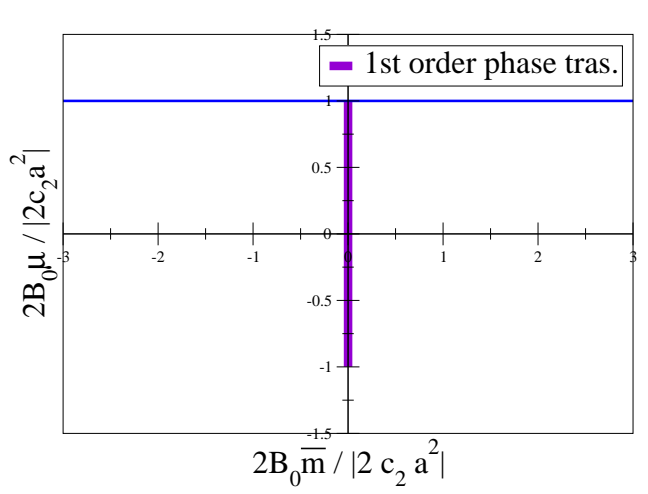

(a) $\tilde{m}$ vs $\mu_{0}$ at $c_{2}<0, \tilde{c}_{2}=0 \llbracket 11$.

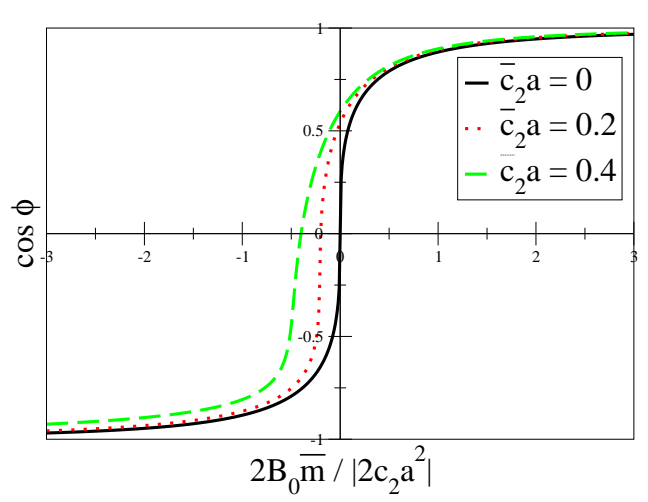

(b) $\tilde{m}$ vs $\cos \phi$ on a blue line of (a).

Figure 1: (a) The phase structure at $c_{2}<0$ and $\tilde{c}_{2}=0$. There is a first order phase transition (violet line), across which $\cos \phi$ jumps. (b) $\cos \phi$ at the end point of first order phase transition, the blue line of (a). A black solid line is the result at $\tilde{c}_{2}=0[11]$, while red dotted and green dashed lines represent results at $\tilde{c}_{2} a=0.2$ and 0.4 , respectively.

\subsection{Pion masses at the LO}

The pion mass at the LO is given by

$$
\begin{aligned}
\left(m_{\pi}^{1,2}\right)^{2} & =2 B_{0} m^{\prime}-2 c_{2} a^{2} \cos ^{2} \phi+2 \tilde{c}_{2} a\left(2 B_{0} m^{\prime}\right) \cos \phi, \\
\left(m_{\pi}^{3}\right)^{2} & =\left(m_{\pi}^{1,2}\right)^{2}+\left(\Delta m_{\pi}^{3}\right)^{2}, \quad\left(\Delta m_{\pi}^{3}\right)^{2}=2 c_{2} a^{2} \sin ^{2} \phi-2 \tilde{c}_{2} a\left(2 B_{0} \mu^{\prime}\right) \sin \phi,
\end{aligned}
$$

where mass terms $m^{\prime}$ and $\mu^{\prime}$ are rotated masses, given by

$$
\left(\begin{array}{c}
m^{\prime} \\
\mu^{\prime}
\end{array}\right)=\left(\begin{array}{cc}
\cos \phi & \sin \phi \\
-\sin \phi & \cos \phi
\end{array}\right)\left(\begin{array}{l}
\tilde{m} \\
\mu
\end{array}\right) .
$$

\section{Next leading order analysis}

\subsection{One-loop calculations}

In our power counting scheme, SLO terms generate three-point vertices. One-loop diagrams are plotted for the vacuum expectation value in Fig 2 a) and for pion propagators in Fig 2 b,c), where the solid (double) line represents the charged (neutral) pion.

At one-loop the vacuum expectation value has divergences, which must be removed by NLO local counter terms. Our counting scheme in the WChPT tell us that one-loop diagrams in Fig 2 a) generates $\mathrm{LO} \times \mathrm{SLO}$ contributions as

$$
\begin{aligned}
\bigcirc= & (\mathrm{SLO}) \int d p^{4} \frac{1}{p^{2}-m_{\pi}^{2}} \\
& =\mathrm{LO} \times \mathrm{SLO},
\end{aligned}
$$

where the three-point vertex is the SLO and $p^{2}$ and $m_{\pi}^{2}$ are the LO.

In the case of pion propagators here exist two types of 1-loop diagrams as shown in Fig 2 b,c). The first type diagrams are made from a four-point vertex, while the second ones are made from 

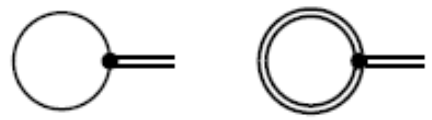

(a) One-loop diagrams for the vacuum expectation value.

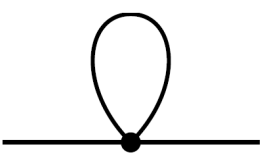

(b) A first type diagram for pion propagators at one-loop

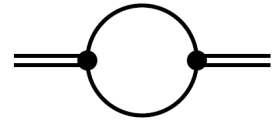

(c) Second type diagram for pion propagators at one-loop

Figure 2: One-loop diagrams for vacuum expectation values (a) and pion propagators (b,c). Diagrams for pion propagators are classified into two types. The first type (b) leads to $\mathrm{LO}^{2}$ effects while the second type (c) leads to $\mathrm{SLO}^{2}$.

two three-point vertices. Since the four-points vertex contains LO terms, the first type diagrams generate $\mathrm{LO}^{2}$ contributions as

$$
\begin{aligned}
\bigcap & =(\mathrm{LO}) \int d p^{4} \frac{1}{p^{2}-m_{\pi}^{2}} \\
& =\mathrm{LO}^{2},
\end{aligned}
$$

while the second type diagrams give $\mathrm{SLO}^{2}$ effects as

$$
\begin{aligned}
\Longrightarrow= & (\mathrm{SLO})^{2} \int d p^{4} \frac{1}{p^{2}-m_{\pi}^{2}} \frac{1}{p^{2}-m_{\pi}^{2}} \\
& =\mathrm{SLO}^{2} .
\end{aligned}
$$

Therefore the second type diagrams are higher order than NLO and we do not consider them in the following analysis.

\subsection{NLO local counter terms}

The NLO Lagrangian is given by,

$$
\begin{aligned}
\mathscr{L}_{\mathrm{NLO}} & =\mathscr{L}_{p^{4}}+\mathscr{L}_{a p^{4}}+\mathscr{L}_{a^{2} p^{2}}, \\
\mathscr{L}_{p^{4}} & =L_{45}\left\langle\Sigma \tilde{M}^{\dagger}+\tilde{M} \Sigma^{\dagger}\right\rangle\left\langle L_{\mu \mu}\right\rangle+L_{68}\left\langle\Sigma \tilde{M}^{\dagger}+\tilde{M} \Sigma^{\dagger}\right\rangle^{2}, \\
\mathscr{L}_{a p^{4}} & =\left\langle L_{\mu \mu}\right\rangle\left(V_{1}\left\langle\Sigma \hat{a}^{\dagger}+\hat{a} \Sigma^{\dagger}\right\rangle\left\langle\Sigma \tilde{M}^{\dagger}+\tilde{M} \Sigma^{\dagger}\right\rangle+V_{2}\left\langle\hat{a} \tilde{M}^{\dagger}+\tilde{M} \hat{a}^{\dagger}\right\rangle\right) \\
& +V_{3}\left\langle\partial_{\mu} \Sigma \hat{a}^{\dagger}+\hat{a} \partial_{\mu} \Sigma^{\dagger}\right\rangle\left\langle\partial_{\mu} \Sigma \hat{a}^{\dagger}+\hat{a} \partial_{\mu} \Sigma^{\dagger}\right\rangle \\
& +V_{4}\left\langle\Sigma \hat{a}^{\dagger}+\hat{a} \Sigma^{\dagger}\right\rangle\left\langle\Sigma \tilde{M}^{\dagger}+\tilde{M} \Sigma^{\dagger}\right\rangle^{2}+V_{5}\left\langle\tilde{M} \tilde{M}^{\dagger}\right\rangle\left\langle\Sigma \hat{a}^{\dagger}+\hat{a} \Sigma^{\dagger}\right\rangle \\
& +V_{6}\left\langle\hat{a} \tilde{M}^{\dagger}+\tilde{M} \hat{a}^{\dagger}\right\rangle\left\langle\Sigma \tilde{M}^{\dagger}+\tilde{M} \Sigma^{\dagger}\right\rangle, \\
\mathscr{L}_{a^{2} p^{2}} & =\left\langle L_{\mu \mu}\right\rangle\left(X_{1}\left\langle\Sigma \hat{a}^{\dagger}+\hat{a} \Sigma^{\dagger}\right\rangle^{2}+X_{2}\left\langle\hat{a} \hat{a}^{\dagger}\right\rangle\right)+X_{3}\left\langle\partial_{\mu} \Sigma \hat{a}^{\dagger}+\hat{a} \partial_{\mu} \Sigma^{\dagger}\right\rangle^{2} \\
& +X_{4}\left\langle\Sigma \hat{a}^{\dagger}+\hat{a} \Sigma^{\dagger}\right\rangle^{2}\left\langle\Sigma \tilde{M}^{\dagger}+\tilde{M} \Sigma^{\dagger}\right\rangle+X_{5}\left\langle\hat{a} \hat{a}^{\dagger}\right\rangle\left\langle\Sigma \tilde{M}^{\dagger}+\tilde{M} \Sigma^{\dagger}\right\rangle \\
& +X_{6}\left\langle\hat{a} \tilde{M}^{\dagger}+\tilde{M} \hat{a}^{\dagger}\right\rangle\left\langle\Sigma \hat{a}^{\dagger}+\hat{a} \Sigma^{\dagger}\right\rangle,
\end{aligned}
$$

where fourteen coefficients $L_{i}, V_{i}, X_{i}$ are new low energy constants.

\subsection{Renormalization}

Calculating one-loop diagram in Fig 2 (a,b), it seems that number of divergent terms is more than the number of local terms, fourteen. Using the gap equation (3.1) rewritten in terms of (3.4) 
as

$$
2 B_{0} \mu^{\prime}=-c_{2} a^{2} \sin 2 \phi+\tilde{c}_{2} a\left(2 B_{0} m^{\prime} \sin \phi-2 B_{0} \mu^{\prime} \cos \phi\right),
$$

we can reduce the number of independently divergent terms as follows.

We first list the coefficients of NLO local terms as

$$
\mathscr{L}_{p^{4}}: m^{\prime 2}, m^{\prime} \mu^{\prime}, \mu^{\prime 2}, \quad \mathscr{L}_{a p^{4}}: a m^{\prime 2}, a m^{\prime} \mu^{\prime}, a \mu^{\prime 2}, \quad \mathscr{L}_{a^{2} p^{2}}: a^{2} m^{\prime}, a^{2} \mu^{\prime}
$$

Using relations among these coefficients derived from equation (4.8), it can be shown that independent coefficients are given by $m^{\prime 2}, a m^{\prime 2}, a^{2} m^{\prime}$, Similarly we can reduce the number of divergence terms of one-loop diagrams, so that the number of independently divergent terms is smaller than that of local counter terms. Therefore we can renormalize all divergences generated from one-loop diagrams by NLO local counter terms in WChPT for tmLQCD.

\section{Maximal twist and pion mass at the NLO}

Physical observables are automatically $O(a)$ improved at the maximal twist, which is realized by tuning the (untwisted) quark mass $\tilde{m}$. Among several definitions proposed so far, we consider the maximal twist defined through the PCAC quark mass [5, 12 in the framework of the WChPT.

\subsection{Maximal twist condition from the PCAC quark mass}

A definition of the maximal twist is given by a condition that the PCAC quark mass vanishes, which is explicitly written as

$$
m_{\mathrm{PCAC}}=\frac{\sum_{\vec{x}}\left\langle\partial_{0} A_{0}^{a}(\vec{x}, t) P^{a}(0)\right\rangle}{2 \sum_{\vec{x}}\left\langle P^{a}(\vec{x}, t) P^{a}(0)\right\rangle}=0, \quad a=1,2
$$

Using the LO Lagrangian, the PCAC quark mass is calculated as

$$
m_{\mathrm{PCAC}}=\frac{\left(m_{\pi}^{a}\right)_{\mathrm{LO}}^{2} \cos \phi}{2 B_{0}\left(1+\tilde{c}_{2} a \cos \phi\right)},
$$

which correctly leads to the the maximal twist condition, $\cos \phi=0$. This condition simplify the gap equation (3.1),

$$
2 B_{0} \tilde{m}=-2 B_{0} \mu \tilde{c}_{2} a .
$$

Eq.(5.3) shows that the critical quark mass $\tilde{m}$ depends linearly on the twisted quark mass $\mu$. This linear dependence has been actually observed in numerical simulations [3. Note however that our analysis here does not contain NLO contributions, which likely give higher order contributions such as $\tilde{m}^{2}, \mu^{2}$ and $\tilde{m} \mu$.

\subsection{Pion mass at maximal twist}

Using eq. (5.3), the charged pion mass at the NLO is given by

$$
\left(m_{\pi}^{1,2}\right)_{\mathrm{NLO}}^{2}=\left(m_{\pi}^{1,2}\right)_{\mathrm{LO}}^{2}\left\{1-\frac{16}{f^{2}}\left(m_{\pi}^{1,2}\right)_{\mathrm{LO}}^{2}\left(2 L_{68}+L_{45}\right)+\frac{1}{f^{2}} a^{2} \mathscr{X}+\frac{3}{4} \frac{\left(m_{\pi}^{3}\right)^{2}}{16 \pi^{2} f^{2}} \ln \left(\frac{m_{\pi}^{3}}{\mu_{\mathrm{ChPT}}}\right)^{2}\right\}
$$


where $\mathscr{X}$ represent a combination of several low energy constants, and LO pion masses $\left(m_{\pi}^{a}\right)_{\mathrm{LO}}^{2}$ are simplified as $\left(m_{\pi}^{1,2}\right)_{\mathrm{LO}}^{2}=2 B_{0} \mu$ and $\left(m_{\pi}^{3}\right)_{\mathrm{LO}}^{2}=2 B_{0} \mu+2 c_{2} a^{2}-2\left(\tilde{c}_{2} a\right)^{2} 2 B_{0} \mu$.

Eq. (5.4) shows that the charged pion mass is $O(a)$ improved as lattice spacing corrections starts at $O\left(a^{2}\right)$, It is interesting to see that the charged pion mass at the NLO contains the chiral log only from neutral pion loops but not from charged pion loops at the maximal twist.

\section{Summary}

We construct the WChPT for the twisted mass lattice QCD at the small quark mass regime such that $m_{q} \sim a^{2} \Lambda^{3}$. Although the non-trivial phase structure and the pion mass splitting at the LO appear to be similar to previous studies, the vacuum expectation value at the NLO has divergences, which can be renormalized by NLO local counter terms with the help of the gap equation 3.1.

As an interesting application of NLO calculations, we consider the $O(a)$ improvement at the maximal twist defined through the PCAC quark mass at the LO. We have found that the critical untwisted quark mass linearly depend on the twisted quark mass and the charged pion mass is $O(a)$ improved at the NLO.

This work was supported in part by the Grant-in-Aid of the Japanese Ministry of Education, Science, Sports and Culture (No. 20340047).

\section{References}

[1] R. Frezzotti, P. A. Grassi, S. Sint and P. Weisz [Alpha collaboration], JHEP 0108, 058 (2001) [arXiv:hep-lat/0101001].

[2] R. Frezzotti, S. Sint and P. Weisz [ALPHA collaboration], JHEP 0107, 048 (2001) [arXiv:hep-lat/0104014].

[3] C. Urbach [European Twisted Mass Collaboration], PoS LAT2007, 022 (2007) [arXiv:0710.1517 [hep-lat]].

[4] $\mathrm{Ph}$. Boucaud et al. [ETM collaboration], arXiv:0803.0224 [hep-lat].

[5] R. Frezzotti and G. C. Rossi, JHEP 0408, 007 (2004) [arXiv:hep-lat/0306014].

[6] S. Aoki and O. Bar, Phys. Rev. D 74, 034511 (2006) [arXiv:hep-lat/0604018].

[7] S. Aoki, Phys. Rev. D 30, 2653 (1984).

[8] S. R. Sharpe and R. L. . Singleton, Phys. Rev. D 58, 074501 (1998) [arXiv:hep-lat/9804028].

[9] S. Aoki, Phys. Rev. D 68, 054508 (2003) [arXiv:hep-lat/0306027].

[10] G. Munster and C. Schmidt, Europhys. Lett. 66, 652 (2004) [arXiv:hep-lat/0311032].

[11] S. R. Sharpe and J. M. S. Wu, Phys. Rev. D 70, 094029 (2004) [arXiv:hep-lat/0407025].

[12] S. Aoki and O. Bar, Phys. Rev. D 70, 116011 (2004) [arXiv:hep-lat/0409006]. 